NASA TECHN ICAL R E P O R T

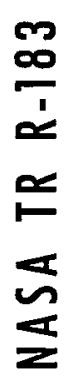

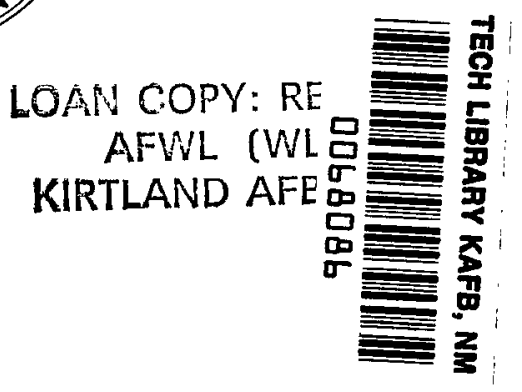

\title{
OBSERVED TORQUE-PRODUCING FORCES ACTING ON SATELLITES
}

by Robert J. Naumann

George C. Marshall Space Flight Center

Huntsville, Ala.

NATIONAL AERONAUTICS AND SPACE ADMINISTRATION - WASHINGTON, D. C. - DECEMBER 1963 
OBSERVED TORQUE-PRODUCING FORCES

ACTING ON SATELLITES

By Robert J. Naumann

George C. Marshall Space Flight Center Huntsville, Ala.

NATIONAL AERONAUTICS AND SPACE ADMINISTRATION

For sale by the Office of Technical Services, Department of Commerce, Woshington, D.C. 20230 -- Price $\$ 1.00$ 


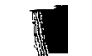

翼 
TABLE OF CONTENTS

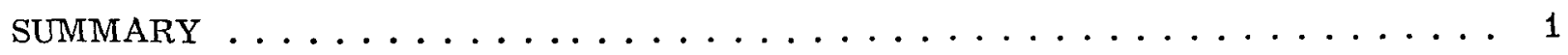

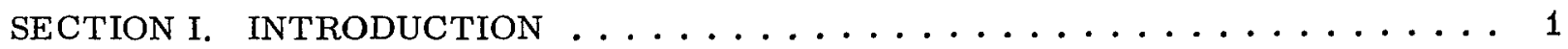

SECTION II. COMPUTATIONAL TECHNIQUES ................ 3

A. Gravitational Gradient Torques ..................... 3

B. Magnetic Torques......................... 5

C. Induced Magnetic Torques ...................... 6

D. Drag Torques .............................. 7

E. Accuracy of Approximations .................... 8

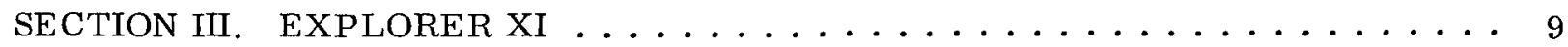

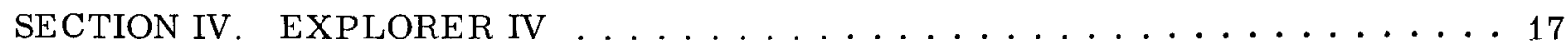

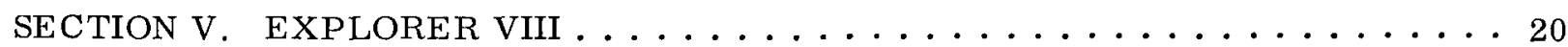

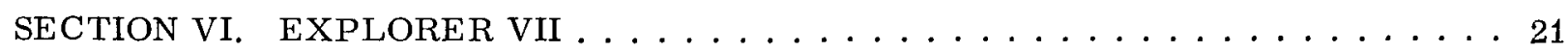

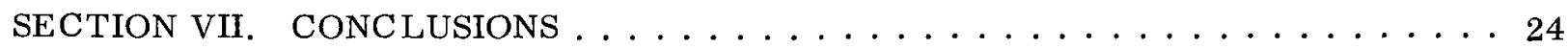




\section{LIST OF ILLUSTRATIONS}

Figure

Title

Page

1. Observed Orientation of the Angular Momentum Vector of

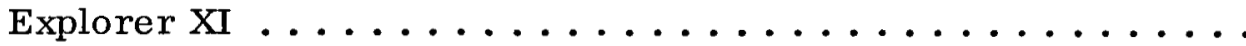

2. Analysis of the Permanent and Induced Magnetic Torques Compared to the Difference Between the Observed Torque and Gravitational Torque for Explorer XI ............

3. Analysis of the Permanent Magnetic and Gravitational Torque Compared to the Observed Torque for Explorer XI ........

4. Computed Orientation of the Angular Momentum Vector Compared to Observations for the First 20 Days of

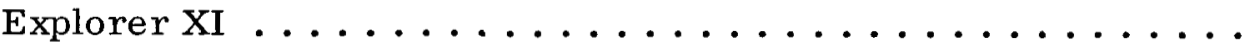

5. Computed Orientation of Explorer XI Compared to Observations for Day $26-90 \ldots \ldots \ldots \ldots \ldots \ldots$

6. Analysis of the Computed Orientation of Explorer IV Due to Various Assumptions Compared to Observation...........

7. Observed Axial Roll Period for Explorer IV . . . . . . . .

8. Computed Solar Aspect Angle for Explorer VIII Compared

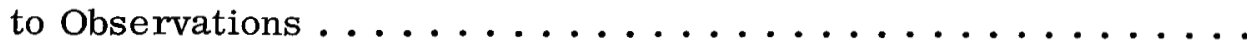

9. Computed Orientation of Explorer VIII .............

10. Computed Orientation of Explorer VII with and without an

Assumed Magnetic Moment ................... 


\section{NATIONAL AERONAUTICS AND SPACE ADMINISTRATION}

\section{TECHNICAL REPORT R-183}

\section{OBSERVED TORQUE-PRODUCING FORCES \\ ACTING ON SATELLITES}

by

Robert J. Naumann

\section{SUMMARY}

In addition to the forces that influence the motion of the center of mass of a satellite, various forces are also present which influence the motion about the center of mass. These torque-producing forces may result in a change in satellite orientation that affects the thermal balance, operation of solar cells, various scientific measurements, and drag forces. This latter effect results in a coupling between the equations describing the orientation with those describing the orbital motion. Therefore, strictly speaking, the orientation problem is part of the orbital problem.

To solve the orientation equations it is necessary to determine the nature of the torque-producing forces. This is done by observing the change in orientation of several Explorer satellites where the drag force is sufficiently small so the orbital dependence on orientation is negligible. It was found that permanent magnetic moments in the satellites were the dominant effect.responsible for the observed changes in orientation. Gravitational torques are also significant. The changes in orientation of the satellites considered were explained extremely well by these two effects, hence it is concluded that other effects are not significant for similar satellites.

Various approximations used in this study greatly reduce the effort required to integrate the orientation equations and do not require the simultaneous solution of the orbital equations. The findings, however, a re applicable to the simultaneous solution of both the orbital and orientation sets of equations.

\section{SECTION I. INTRODUCTION}

The most general treatment of satellite motion requires six second-order differential equations to describe the motion. Three equations describe the motion of the center 
of mass in phase space, and the remaining three equations describe the orientation and rates of three body-fixed axes relative to a space-fixed system. In orbital calculations it is usually assumed that this set of six equations separates into two sets with the orbital set independent of the set describing the orientation. This is not rigorously true if the satellite in question has any asymmetry, since the drag forces will depend on the orientation of the satellite.

It is desirable for several reasons to formulate the full six degree of freedom problem and integrate the equations describing the orientation along with the orbital equations. It may be argued that since the previously mentioned drag dependence is usually small compared to the uncertainties in atmospheric density, such an exercise is largely academic. While this is a valid argument there are many satellite experiments in which it is just as important, or even more important, for the experimenter to have knowledge of the satellite orientation as it is to know the position of the center of mass. This is particularly true in the case of the Explorer XI gamma ray astronomy satellite.

In most cases where it is necessary to have orientation information, the approach has been to derive such information from a combination of solar and earth horizon sensors contained in the satellite, or by analyzing the recorded radio signal strength patterns. The latter has been particularly successful with Explorers IV, VII, and XI.

While it has been possible to determine satellite orientation a posteriori, it would be desirable to be able to predict the satellite orientation a priori. Since the external torque-producing forces are coupled closely to the motion of the center of mass, and since the motion of the center of mass depends to a lesser degree on the orientation, it would seem reasonable to consider the entire six degree of freedom formulation in the integration of the equations of motion. A convenient formulation of the full six degree problem has been suggested by Lundquist and Naumann [Ref. 1] in which the orientation of the body-fixed axes are expressed in terms of the Euler quaternion parameters [Ref. 2]. This formulation has several advantages over the more conventional Euler angle formulation in that it yields symmetrical equations in which no trigonometric functions are involved. These parameters also have certain advantages over the CaleyKlein parameters [Ref. 3] in that complex numbers are not involved. The disadvantage is that they are not independent and exceed by one the number of degrees of freedom. This difficulty is alleviated by the use of a Lagrange undetermined multiplier and the constraint relationship that the sum of the squares of the parameters is unity. Several computer programs based on this formulation have been developed by the Computation Division at the Marshall Space Flight Center and by Cunningham at the University of California.

In order to use such programs to predict satellite motion about the center of mass, it is necessary to know the nature of the external torques acting on the satellite. Analysis of the motion of several of the Explorer satellites has yielded some knowledge concerning the nature of these torques. The purpose of this paper is to discuss some of the 
experimental satellite orientation data, and attempt to explain the nature and origin of the torques responsible for the observed motion about the center of mass.

The satellites to be considered are Explorer XI, Explorer IV, Explorer VIII and Explorer VII. This particular order was chosen because Explorer XI has yielded the most accurate and extensive orientation information.. Sufficient information has been obtained to allow the extraction of the torques by numerical differentiation, hence, the determination of significant effects. Much less orientation data are available for Explorer IV, but in the light of the Explorer XI analysis, its motion is now fairly well understood. Very little data exist on Explorers VIII and VII, but again using results from the previous analyses a motion is derived that is consistent with known data.

\section{SECTION II. COMPUTATIONAL TECHNIQUES}

Before discussing the various satellites individually, it would be well to discuss various sources of torques and the various approximations used in computing their effects.

To begin, it will be assumed that all of the satellites have cylindrical symmetry and a re initially set spinning about their axes of symmetry. The moment of inertia about the axis of symmetry is denoted as $\mathrm{I}_{3}$. . If a transverse axis has a moment of inertia $I_{2}$ less than $I_{3}$, the satellite is said to be stable and will remain spinning about $I_{3}$. If $I_{2}$ is greater than $I_{3}$, as is the case in Explorer $X I$ and Explorer $I V$, the satellite is in a maximum rotational energy configuration and is unstable. Due to internal energy dissipation and angular momentum conservation, the satellite longitudinal axis will precess about the angular momentum vector in a cone. The half-angle of this precession cone $\alpha$ is related to the longitudinal spin $\omega_{3}$ by

$$
\cos \alpha=\frac{\mathrm{I}_{3} \omega_{3}}{\mathrm{~L}}
$$

where $L$ is the total angular momentum. As energy is dissipated, $\omega_{3} \rightarrow 0, \alpha \rightarrow \pi / 2$, and the satellite approaches the minimum energy configuration in which the rotation is a tumbling motion with the axis of symmetry rotating in a plane perpendicular to the angular momentum vector.

\section{A. GRAVITATIONAL GRADIENT TORQUES}

The gravitational torque on a satellite may be found by expanding the potential energy about the center of mass in a Taylor series and differentiating with respect to the generalized angles expressing the orientation of the body-fixed axes. The result given by Roberson [Ref. 4] and others [Ref. 5] is 


$$
\overrightarrow{\mathrm{L}}_{\mathrm{G}}=3 \frac{\mathrm{mk}^{2}}{\mathrm{r}^{3}}\left(\mathrm{I}_{1}-\mathrm{I}_{3}\right) \quad(\hat{\mathrm{A}} \cdot \hat{\mathrm{R}}) \quad(\hat{\mathrm{A}} \times \hat{\mathrm{R}})
$$

where $\mathrm{mk}^{2}$ is the gravitational constant, $\mathrm{r}$ is the radius vector magnitude, $\hat{A}$ is a unit vector along the axis of cylindrical symmetry, and $\hat{R}$ is a unit vector along the radius vector.

Assuming a half-cone angle $\alpha$, this torque may be time averaged over a precessional or tumble cycle while holding $\hat{\mathrm{R}}$ constant. This results in

$$
\overrightarrow{\mathrm{L}}_{\mathrm{G}}=\frac{3}{2} \frac{\mathrm{mk}^{2}}{\mathrm{r}^{3}}\left(\mathrm{I}_{1}-\mathrm{I}_{3}\right)(\hat{\mathrm{R}} \cdot \hat{\mathrm{L}})(\hat{\mathrm{R}} \times \hat{\mathrm{L}})\left(1-3 \cos ^{2} \alpha\right)
$$

where $\hat{\mathrm{L}}$ is a unit vector directed along the angular momentum vector.

Assuming a circular orbit, this $\overrightarrow{\mathrm{L}}_{\mathrm{G}}$ is time averaged over an orbital revolution while holding $\hat{L}$ constant. This is done by expressing $\hat{R}$ in terms of orbital inclination $i$, right ascension of the ascending node $\phi$, and mean anomaly $M$.

$$
\hat{\mathbf{R}}=\left(\begin{array}{cc}
\cos M & \cos \phi-\cos i \sin \phi \sin M \\
\cos M & \sin \phi+\cos i \cos \phi \sin M \\
& \sin i \sin M
\end{array}\right)
$$

The unit vector along the orbital angular momentum is

$$
\hat{\Omega}=\left(\begin{array}{c}
\sin i \sin \phi \\
-\sin i \cos \phi \\
\cos i
\end{array}\right)
$$

The time average is found by

$$
\overrightarrow{\mathrm{L}}_{\mathrm{G}}=\frac{3}{4 \pi} \frac{\mathrm{mk}^{2}}{\mathrm{r}^{3}}\left(\mathrm{I}_{2}-\mathrm{I}_{3}\right)\left(1-3 \cos ^{2} \alpha\right) \int_{0}^{2 \pi}(\hat{\mathrm{R}}: \hat{\mathrm{L}})(\hat{\mathrm{R}} \times \hat{\mathrm{L}}) \mathrm{dM} .
$$

Expressing $\hat{\mathrm{R}}$ in terms of $\phi$, $\mathrm{i}$, and $\mathrm{M}$ from equation 4 , integrating, and expressing the resulting combinations of $\phi$ and $i$ in terms of components of $\hat{\Omega}$ using equation 5 , results in 


$$
\overrightarrow{\mathrm{L}}_{\mathrm{G}}=\mathrm{K}_{\mathrm{G}}(\hat{\mathrm{L}} \cdot \hat{\Omega})(\hat{\mathrm{L}} \times \hat{\Omega})\left(1-3 \cos ^{2} \alpha\right),
$$

where

$$
\mathrm{K}_{\mathrm{G}}=\frac{3}{4} \frac{\mathrm{mk}^{2}}{\mathrm{r}^{3}}\left(\mathrm{I}_{1}-\mathrm{I}_{3}\right) .
$$

\section{B. MAGNETIC TORQUES}

If a satellite has a permanent magnetic moment projection, $\mathrm{M}_{\mathrm{L}}$, on the axis of rotation, an interaction torque will arise from the geomagnetic field. This torque is given by

$$
\overrightarrow{\mathrm{L}}_{M}=\overrightarrow{\mathrm{M}}_{L} \times \overrightarrow{\mathrm{B}}=\mathrm{M}_{L}(\hat{\mathrm{L}} \times \hat{\mathrm{B}})
$$

A component of magnetic moment along the axis of symmetry, $\mathrm{M}_{3}$, results in $\mathrm{M}_{\mathrm{L}}=\mathrm{M}_{3} \cos \alpha$. Due to rotational symmetry, a transverse component of magnetic moment does not contribute unless $\omega_{3}=0$. If this happens, the $\mathrm{M}_{\mathrm{L}}$ is $\mathrm{M}_{1}$ or the projection of the transverse component on $\hat{\mathrm{L}}$.

To facilitate computation, an earth-centered dipole representation of the geomagnetic field is employed,

$$
\vec{B}=B_{N}\left(\begin{array}{c}
-3 R_{x} R_{z} \\
-3 R_{y} R_{z} \\
1-3 R_{z}^{2}
\end{array}\right) \delta
$$

where $B_{N}$ is the scalar field at the equator at $r \mathrm{~km}$ from the center of the earth. This is given by

$$
\mathrm{B}_{\mathrm{N}}=\frac{\gamma}{\mathrm{r}^{3}}
$$

where $\gamma$ is the geomagnetic moment taken as $8.1 \times 10^{25} \mathrm{cgs}$ units.

Assuming a circular orbit, the magnetic torque is time averaged over an orbital revolution in the same manner as before, giving 


$$
\overrightarrow{\mathrm{L}}_{\mathrm{M}}=\mathrm{M}_{\mathrm{L}} \mathrm{B}_{\mathrm{N}}\left(\begin{array}{c}
-\frac{1}{2} \mathrm{~L}_{\mathrm{y}}+\frac{3}{2} \Omega_{\mathrm{z}}(\hat{\mathrm{L}} \times \hat{\Omega})_{\mathrm{x}} \\
\frac{1}{2} \mathrm{~L}_{\mathrm{x}}+\frac{3}{2} \Omega_{\mathrm{z}}(\hat{\mathrm{L}} \times \hat{\Omega})_{\mathrm{y}} \\
\frac{3}{2} \Omega_{\mathrm{z}}(\hat{\mathrm{L}} \times \hat{\Omega})_{\mathrm{z}}
\end{array}\right)
$$

The constant $M_{L} B_{N}$ is termed the magnetic couple.

\section{INDUCED MAGNETIC TORQUES}

For a satellite having a long cylindrical geometry, it is possible that a magnetic moment may be induced that alternates in sign during a tumble cycle [Ref. 6]. Torques produced by this process would tend to add rather than cancel because of rotational symmetry. The induced moment is

$$
\overrightarrow{\mathrm{M}}_{\mathrm{I}}=\frac{\mu_{\mathrm{r}}-1}{\mu_{\mathrm{O}}} \mathrm{U}(\overrightarrow{\mathrm{B}} \cdot \hat{\mathrm{A}}) \hat{\mathrm{A}} \quad \text { (rationalized MKS units) }
$$

where $\mu_{r}$ is the relative permeability; $\mu_{0}$ is the permeability of free space; and $U$ is the volume of material in the walls of the cylinder. Let

$$
\mathrm{K}_{\mathrm{I}}=\frac{\mu_{\mathrm{r}-1}}{\mu_{\mathrm{o}}} \mathrm{U}
$$

then the torque is

$$
\overrightarrow{\mathrm{L}}_{I}=\mathrm{K}_{\mathrm{I}}(\hat{\mathrm{A}} \cdot \overrightarrow{\mathrm{B}})(\hat{\mathrm{A}} \times \overrightarrow{\mathrm{B}})
$$

Time averaging over a tumble cycle results in

$$
\overrightarrow{\mathrm{L}}_{\mathrm{I}}=-\frac{1}{2} \mathrm{~K}_{\mathrm{I}}(\hat{\mathrm{L}} \cdot \overrightarrow{\mathrm{B}})(\hat{\mathrm{L}} \times \overrightarrow{\mathrm{B}})
$$

Time averaging over an orbital revolution, as before, results in the expressions 


$$
\overrightarrow{\mathrm{L}}_{\mathrm{I}}=-\frac{1}{2} \mathrm{~K}_{\mathrm{I}} \mathrm{B}_{\mathrm{N}}^{2}(\hat{\mathrm{L}} \times \overrightarrow{\mathrm{G}})
$$

where

$$
\vec{G}_{i} \Rightarrow B_{i j} L_{j} ; i=1,2,3 ; j=1,2,3
$$

where

$$
\mathrm{L}_{1}=\mathrm{L}_{\mathrm{x}}, \mathrm{L}_{2}=\mathrm{L}_{\mathrm{y}}, \quad \mathrm{L}_{3}=\mathrm{L}_{\mathrm{z}}
$$

and

$$
\mathrm{B}_{\mathrm{ij}}=\left(\begin{array}{ccc}
\frac{9}{8}\left(\Omega_{\mathrm{y}}^{2}+3 \Omega_{\mathrm{x}}^{2} \Omega_{\mathrm{y}}^{2}\right) & -\frac{9}{8} \Omega_{\mathrm{x}} \Omega_{\mathrm{y}}\left(1-3 \Omega_{\mathrm{z}}^{2}\right) & \frac{3}{8} \Omega_{\mathrm{x}} \Omega_{\mathrm{z}}\left(4-9 \beta^{2}\right) \\
-\frac{9}{8} \Omega_{\mathrm{x}} \Omega_{\mathrm{y}}\left(1-3 \Omega_{\mathrm{z}}^{2}\right) & \frac{9}{8}\left(\Omega_{\mathrm{x}}^{2}+3 \Omega_{\mathrm{y}}^{2} \Omega_{\mathrm{z}}^{2}\right) & \frac{3}{8} \Omega_{\mathrm{y}} \Omega_{\mathrm{z}}\left(4-9 \beta^{2}\right) \\
\frac{3}{8} \Omega_{\mathrm{x}} \Omega_{\mathrm{z}}\left(4-9 \beta^{2}\right) & \frac{3}{8} \Omega_{\mathrm{y}} \Omega_{\mathrm{z}}\left(4-9 \beta^{2}\right) & 1-3 \beta^{2}+\frac{27}{8} \beta^{4}
\end{array}\right)
$$

where

$$
\beta^{2}=\Omega_{\mathrm{x}}^{2}+\Omega_{\mathrm{y}}^{2}
$$

D. DRAG TORQUES

The drag force on a satellite is given by

$$
\overrightarrow{\mathrm{F}}_{\mathrm{D}}=-\frac{1}{2} \cdot \mathrm{C}_{\mathrm{D}} \rho \mathrm{S} \mathrm{V} \overrightarrow{\mathrm{V}}
$$

where $C_{D}$ is the drag coefficient usually taken to be $2.0, \rho$ is the atmospheric density, and $S$ is the area projected normal to the velocity vector $\vec{V}$. The drag torque is

$$
\overrightarrow{\mathrm{i}}_{\mathbf{D}}=\overrightarrow{\mathrm{a}} \times \overrightarrow{\mathrm{F}}_{\mathbf{D}}
$$

where $\vec{a}$ is the displacement of the center of pressure from the center of mass. 
For symmetrical satellites, such as Explorers VII and VIII, the $\vec{a}$ is for all practical purposes zero and the drag torque is not significant. For asymmetrical satellites, such as Explorer XI, the drag torque is significant only before the satellite has reached the tumbling mode. The first order effects cancel by rotational symmetry after $\alpha$ becomes $\pi / 2$.

For an eccentric orbit, such as Explorer XI, the atmospheric density is significant only near perigee. Hence, the $\vec{V}$ is taken as the perigee velocity vector. The time average torque over an orbital revolution is found by

$$
\overrightarrow{\mathrm{L}}_{D}=-\mathrm{K}_{\mathrm{D}}\left(\hat{\mathrm{L}}_{\mathrm{D}} \times \hat{\mathrm{V}}_{\mathrm{P}}\right)
$$

where

$$
\mathrm{K}_{\mathrm{D}}=\frac{1}{2} \mathrm{C}_{\mathrm{D}} \mathrm{S} \mathrm{V} \cdot \mathrm{a} \bar{\rho}
$$

and $\bar{\rho}$ is the time average atmospheric density given by

$$
\bar{\rho}=\frac{1}{2 \pi} \int_{0}^{2 \pi} \rho(\mathrm{r}) \mathrm{d} \mathrm{M} .
$$

\section{E. ACCURACY OF APPROXIMATIONS}

In order to determine the errors introduced in assuming circular orbits and the earth-centered dipole representation for the geomagnetic field, numerical integrations were carried out over actual orbits of Explorer XI, using the Finch and Leaton 48-term Legendre Polynomial representation for the magnetic field [Ref. 7].

It was found that the gravitational torques computed using the approximate methods differed from those computed by integration over an entire orbit by a maximum of $1 \%$.

The permanent magnetic torques differed by approximately $10 \%$ using a value of $\mathrm{B}_{\mathrm{N}}$ of .191 gauss computed from equation 11. However, it was noted that the values obtained from the numerical integration were always larger than those from the approximate method. Assuming a $\mathrm{B}_{\mathrm{N}}$ of . 205 gauss reduced this difference to approximately $\pm 3 \%$. This is not serious in this analysis since the $M_{L}$ is not known accurately and the product $\mathrm{B}_{\mathrm{N}} \mathrm{M}_{\mathrm{L}}$ must be assumed.

The computation of the induced torques suffers somewhat more from the approximations. The maximum observed deviation was $14 \%$ of the maximum value, but 
generally the deviations were no more than $\pm 8 \%$ of the maximum value. The spread was such that no apparent improvement could be gained by slightly altering the value of $\mathrm{B}_{\mathrm{N}}$.

\section{SECTION III. EXPLORER XI}

The orientation history of Explorer XI was obtained by determining the angle a line from the tracking station to the satellite makes with angular momentum vector by analyzing the recorded radio signal strength variations [Ref. 8]. Many such angles with their respective line of sight vectors can be determined during a set of successive passes. These data result in an overdetermined set of non-linear equations that are solved by an iterative process to yield the angular momentum unit vector that best fits the observed data in the sense of least squares. The observed orientation is plotted in terms of right ascension and declination in Figure 1.

Beginning at day 35 after 27. 0 April 1961, these plots are fitted in segments by polynomials which are then differentiated with respect to time to yield the torques responsible for the motion.

Since the gravitational torques can be computed accurately from the known moments of inertia of the satellite (Table 1), they are subtracted from the observed torques and the results are plotted in Figure 2. This resulting torque must then be caused by either a permanent or induced magnetic moment. The induced torques are computed from equation 12 assuming a value of -384 dyne-cm for $\frac{1}{2}-K_{I} B_{N}^{2}$ which was chosen to yield the approximate observed amplitude. It may be seen trom Figure 2 that the induced torques have the same general shape as the required torques, but the correlation is not very convincing. A further difficulty arises in the negative sign that must be assumed for $K_{I}$ since $K_{\text {}}$ should be positive. Several points are shown that were computed from the detailed integration procedure. It is obvious that the errors introduced in the approximations are small compared to the differences between the induced torques and the required torques.

On the other hand, the points resulting from an assumed value of 148.14 dyne-cm for the permanent magnetic couple $M_{L} B_{N}$ fall right on the plots of the required torques. Therefore, it is concluded that a permanent transverse magnetic moment is the dominant effect responsible for the observed motion.

The observed torques together with the gravitational and permanent magnetic torques are plotted in Figure 3 for day 35 through day 180 . It is seen that the sum of gravitational and magnetic torques equals almost exactly the observed torques, the residuals being generally less than five dyne-cm. It is believed that the errors in 


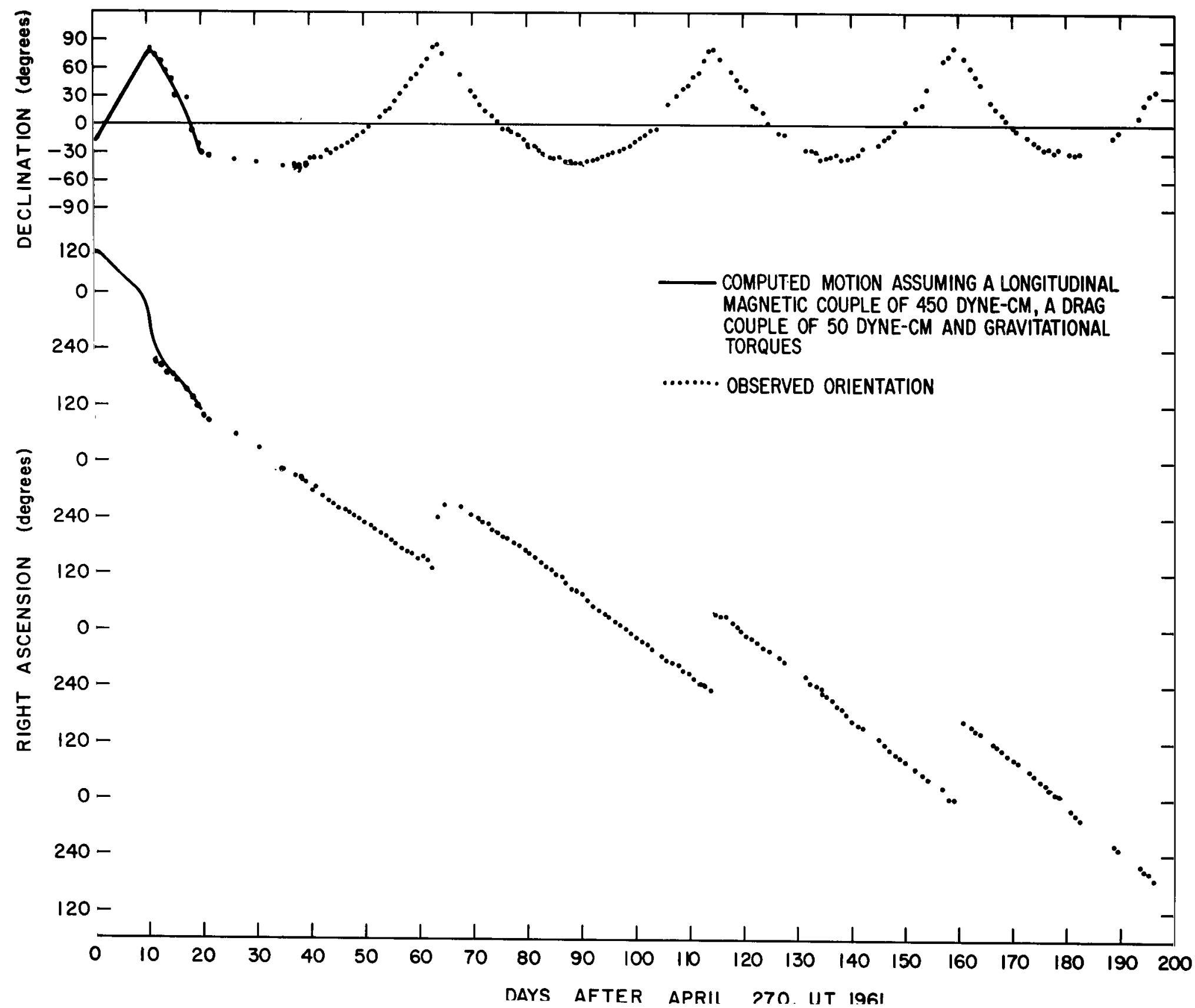

FIGURE 1. OBSERVED ORIENTATION OF THE ANGULAR MOMENTUM VECTOR OF EXPLORER XI 
TABLE 1

\section{DYNAMICAL PROPERTIES}

\section{QUANTITY}

Time Origin

$\mathrm{I}_{1}\left(\mathrm{gm}-\mathrm{cm}^{2}\right)$

$\mathrm{I}_{3}\left(\mathrm{gm}-\mathrm{cm}^{2}\right)$

$\mathrm{I}_{1}-\mathrm{I}_{3}\left(\mathrm{gm}-\mathrm{cm}^{2}\right)$

$\mathrm{r}(\mathrm{km})$

$\mathrm{K}_{\mathrm{G}}($ dyne-cm)

$\mathrm{M}_{1} \mathrm{~B}_{\mathrm{N}}$ (dyne-cm)

$\mathrm{M}_{3} \mathrm{~B}_{\mathrm{N}}$ (dyne-cm)

$\mathrm{M}_{1}\left(\mathrm{amp}-\mathrm{m}^{2}\right)$

$\mathrm{M}_{3}\left(\mathrm{amp}-\mathrm{m}^{2}\right)$

$\phi_{0}(\mathrm{deg})$

$\dot{\phi}$ (deg/day)

i (deg)

$\mathrm{L}\left(\mathrm{gm} \mathrm{cm}^{2} / \mathrm{sec}\right)$

$\Delta \mathrm{L} /$ day

\section{EXPLORER XI}

27. 0 Apr 1961

1. $627 \times 10^{8}$

$0.040 \times 10^{8}$

$1.587 \times 10^{8}$

7512

111.92

148.14

450

.7756

2. 35

253. 912

$-5.0036$

28.8

1. $276 \times 10^{8}$

$-0.223 \times 10^{7 *}$

$-0.0143 \times 10^{7}$
EXPLORER IV

26. 0 Jul 1958

$4.752 \times 10^{7}$

$0.056 \times 10^{7}$

4. $696 \times 10^{7}$

7616

31.78

N/A

$-3500$

N/A

$-19.125$

65.81

$-3.6505$

51.0

4. $568 \times 10^{7}$

$-0.0076 \times 10^{7}$

\section{EXPLORER VII}

13. 0 Oct 1959

$1.08 \times 10^{7}$

$2.93 \times 10^{7}$

$-1.85 \times 10^{7}$

7191

$-14.87$

$\mathrm{N} / \mathrm{A}$

75

$\mathrm{N} / \mathrm{A}$

.345

149. 217

$-4.1745$

50.27

1. $104 \times 10^{9}$

N/A

\section{EXPLORER VIII}

3. 0 Nov 1960

2. $162 \times 10^{7}$

2. $936 \times 10^{7}$

$-0.774 \times 10^{7}$

6964

$-6.85$

N/A

$-200$

N/A

$-.837$

16. 356

$-3.3698$

49.98

$3.073 \times 10^{8}$

$-0.032 \times 10^{7}$

* for first 20 days before tumbling commenced 

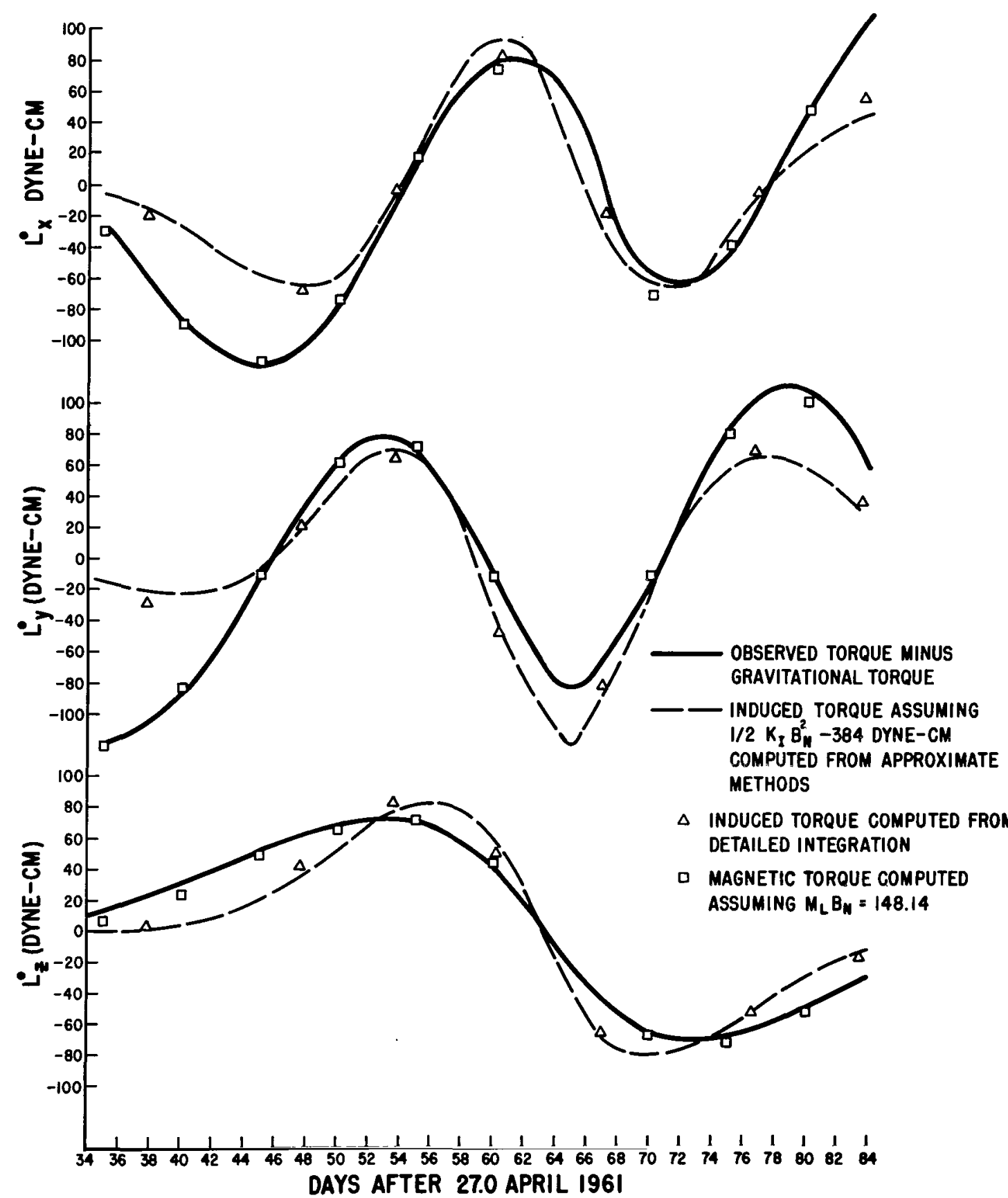

FIGURE 2. ANALYSIS OF THE PERMANENT AND INDUCED MAGNETIC TORQUES COMPARED TO THE DIFFERENCE BETWEEN THE OBSERVED TORQUE AND GRAVITATIONAL TORQUE FOR EXPLORER XI 


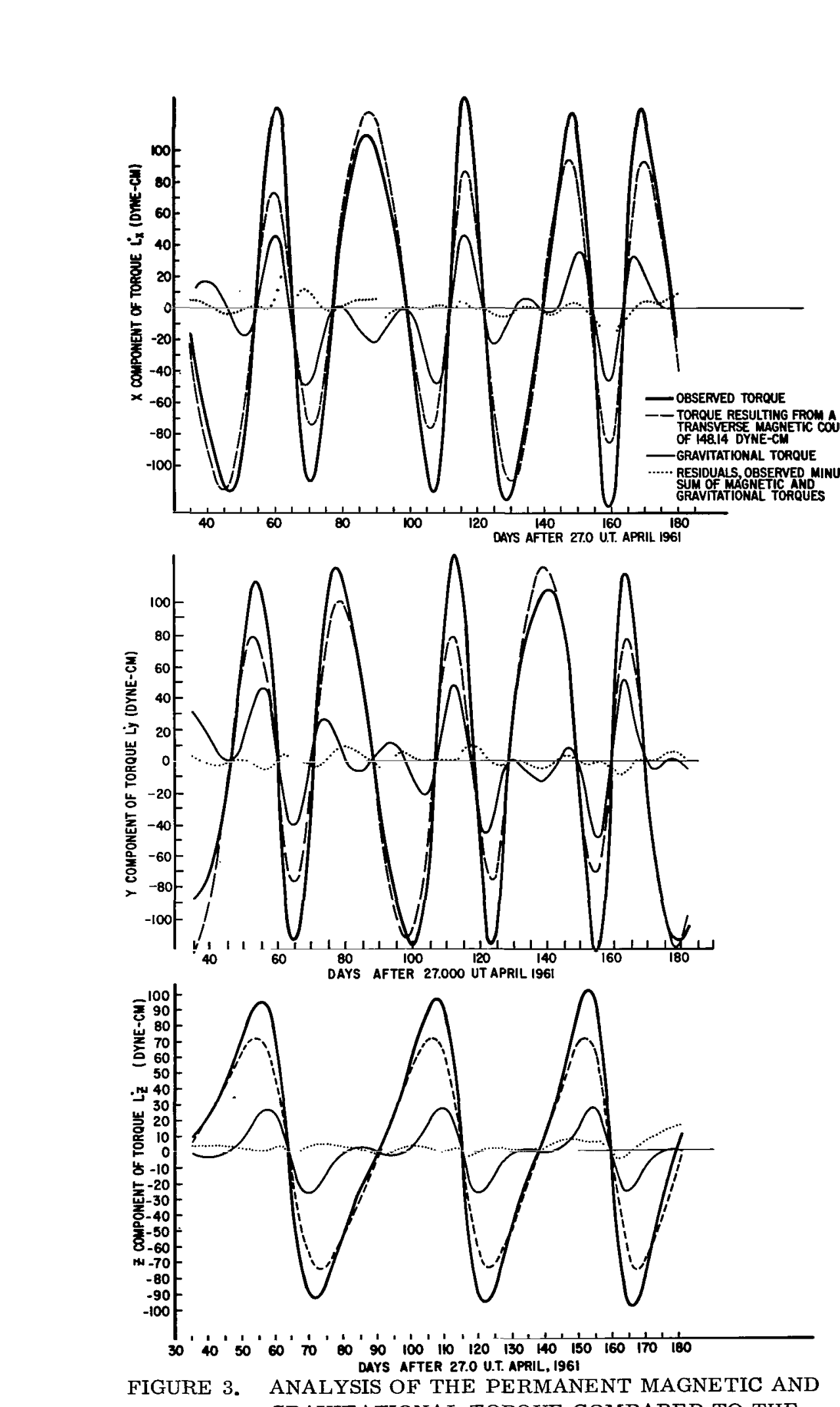


obtaining the torques from the differentiation of the fitted observations are primarily responsible for these deviations, since the greatest deviations usually occur near the ends of the fitted segments.

It is known from solar cell output data that Explorer XI had no $\omega_{3}$ after day 26. This probably occurred because one transverse axis has a slightly greater moment of inertia; hence, there is a preferred axis of tumble: Also, a mercury toroidal damper near the tail is quite effective in removing any residual roll about the longitudinal axis. Therefore, it is quite likely that a transverse magnetic moment can have a constant projection on $\mathrm{L}$.

There is also evidence that a transverse magnetic moment did indeed exist in the attached stainless steel rocket case. Handling difficulties with the loaded rocket casing prevented an accurate measurement of the magnetic moments, but a small hand compass held near the case showed evidence of a transverse as well as a longitudinal magnetic moment.

Although it has been shown that the permanent magnetic moment is the dominant effect, it is still necessary to estimate the magnitude of the induced effect. This is extremely difficult to do in a meaningful manner, since the relative permeability is not well known. In ferrous materials, the permeability depends strongly on the applied field as well as on the past magnetic history of the material. Taking the volume of the ferrous material in the walls of the rocket casing to be $0.470 \times 10^{-3} \mathrm{~m}^{3}$ (the payload portion is fabricated from aluminum) with a permeability of 100 in a field of $0.2 \times 10^{-4}$

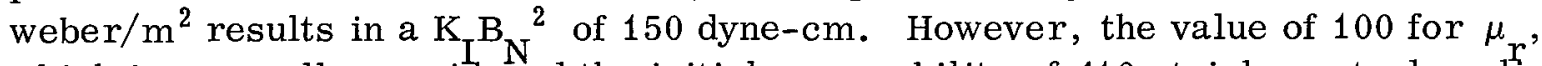
which is generally considered the initial permeability of 410 stainless steel applies for a flux density of 200 gauss in the material. It is probably considerably less for an applied field of \pm 0.2 oersted experienced in the geomagnetic field. There may well be a small component of induced torque, but this would act in the same general manner as the torque due to the permanent magnetism, but with opposite sign. This would probably be compensated for by a slightly greater $M_{L}$ than was assumed.

Since it appears that the gravitational and permanent magnetic torques are mainly responsible for the motion, an attempt was made to supplement the few orientation data available between launch and day 20 before the satellite began tumbling. This was done by starting at the injection orientation and integrating the motion, assuming various values of $M_{3}$ to see if a motion that was consistent with the observed data could be made to result. It was found that a couple of $\mathrm{M}_{3} \mathrm{~B}_{\mathrm{N}}=450$ dyne-cm gave a reasonable fit of the observed orientations. The addition of a drag couple of 50 dyne-cm further improved the fit. The results are shown in Figure 4.

Also, the motion was integrated from day 26 to day 90 and compared with observed data. As may be seen in Figure 5, there is excellent agreement between computed and observed results. 


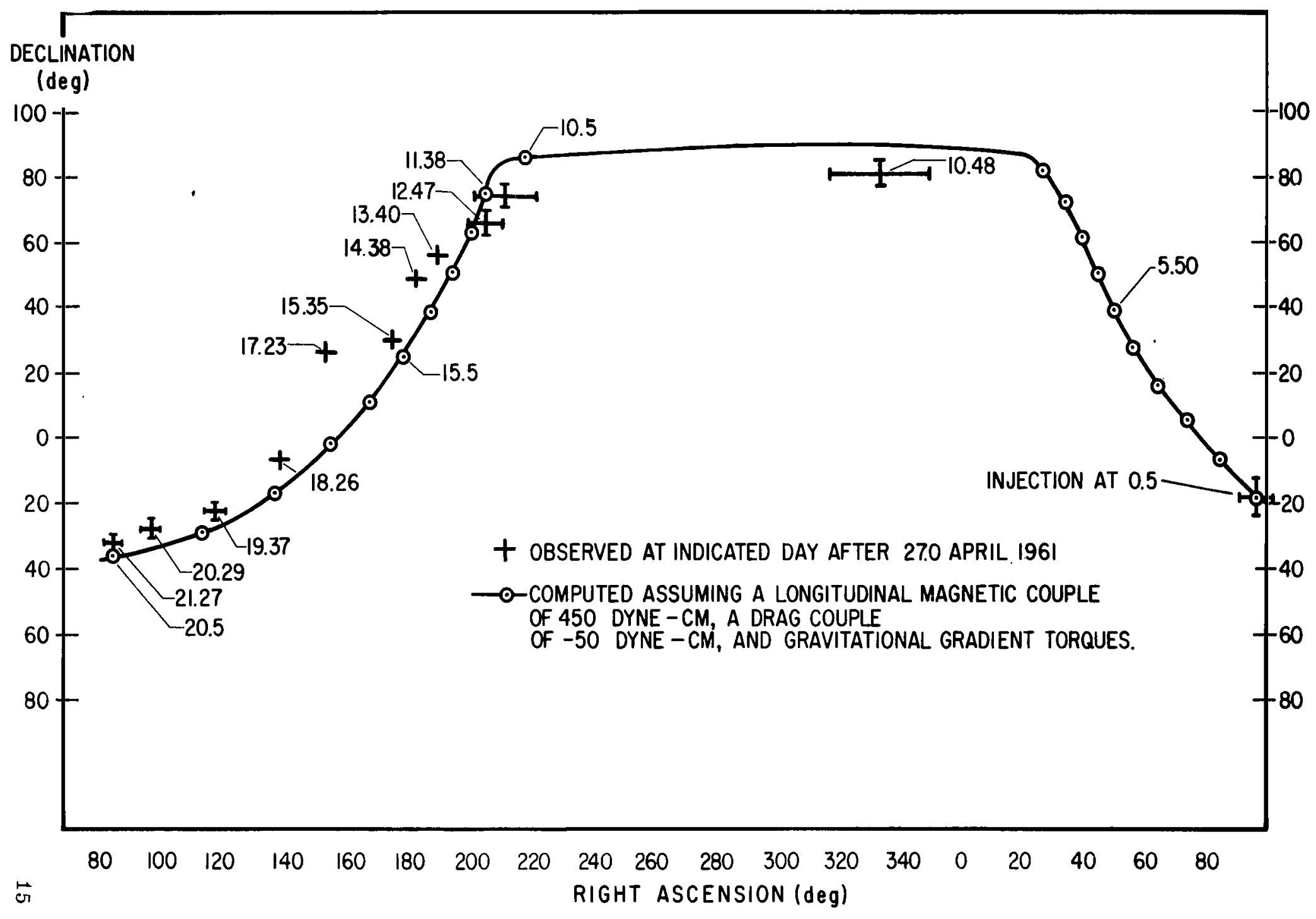

FIGURE 4. COMPUTED ORIENTATION OF THE ANGULAR MOMENTUM VECTOR COMPARED TO OBSERVATIONS FOR THE FIRST 20 DAYS OF EXPLORER XI 


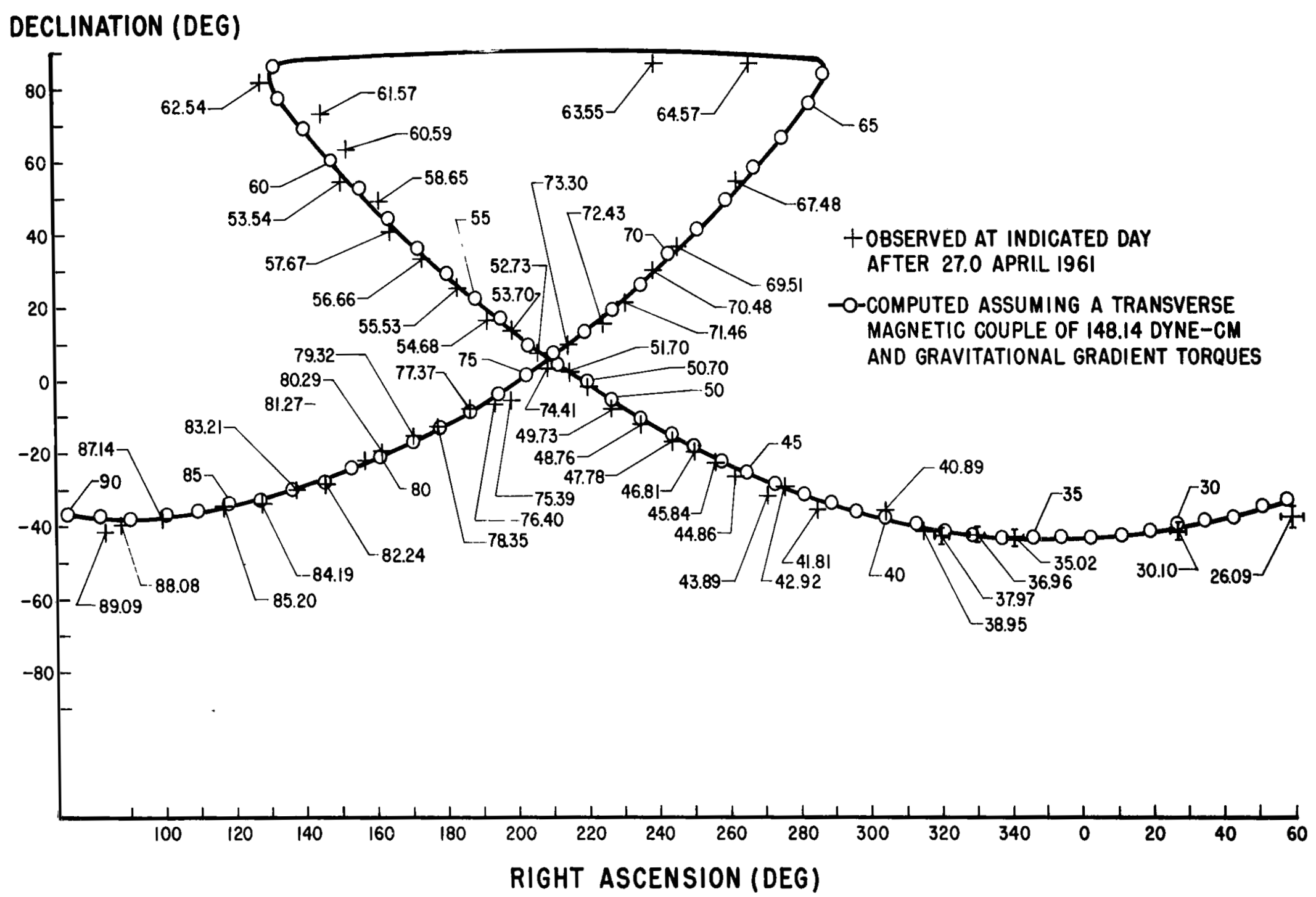

FIGURE 5. COMPUTED ORIENTATION OF EXPLORER XI COMPARED TO OBSERVATIONS FOR DAY 26 - 90 


\section{SECTION IV. EXPLORER IV}

The orientation of Explorer IV was deduced in much the same manne $\mathrm{r}$ as Explorer $\mathrm{XI}$, except that in Explorer IV this analysis was done as an afterthought. Therefore, the choice of tracking antenna and recording techniques was far from optimum for such an analysis. As a result, the amount and accuracy of the orientation data a re much less than for Explorer XI. The data were sufficient to explain the temperature measurements in terms of area presented to the sun [Ref. 9]. Also, the motion of a directional scintillation counter relative to the magnetic field was deduced [Ref. 10] which allowed a determination of the directional flux of the trapped corpuscular radiation in the radiation belts [Ref. 11]. The orientation measurements are shown in Figure 6. It appears that the orientation changes at approximately the same rate as observed in Explorer XI. However, the torques responsible for this motion remained unexplained until quite recently. The possibility of induced magnetic torques was suggested by Colombo [Ref. 12], but starting at day 32. 0 after 26. 0 July 1958 and integrating the motion produced by gravitational and induced torques resulted in a motion that is not consistent with the observed data shown by the short dashed line in Figure 6 .

Integrating the torques produced by a permanent tranverse magnetic couple of -100 dyne-cm, similar to that found in Explorer XI, produced a motion that apparently matches the observed data for several days before and after 32.0. A serious difficulty arises with this assumption, however, since it was known that Explore $f$ IV had a residual $\omega_{3}$ which would tend to cancel any effect due to a transverse magnetic moment. Evidence for this roll is seen as modulations in the observed count rate of a directional scintillation detector whose axis is directed along a transverse axis [Ref. 13]. Figure 7 shows the roll period history deduced from these count rate modulations. Note that the roll period increases rapidly after day 30 . In Figure 6, the change in orientation seems to be much less after this time. From the roll period data, it can be determined that the half-cone angle $\alpha$ increased rapidly the first few days, but approached $\pi / 2$ very slowly. In fact, from equation 1 it may be shown that $\alpha$ is about $83^{\circ}$ on day 30 . It then becomes clear that a longitudinal magnetic moment will give a constant projection on $\hat{\mathrm{L}}$ that will produce the same effect as a transverse magnetic moment. A rather large value of -3500 dyne-cm must be assumed for this couple to give a projected component sufficient to account for the motion between day 25 and day 32 , but this assumption appears to explain the observed data rather well. After day 32 , the torques from gravitational effects appear to be sufficient to explain the motion in a reasonable manner.

There may also be some significant second-order torques produced by the large longitudinal magnetic moment after day 32. These second-order torques arise from the fact that the geomagnetic field is changing because of geographical changes of the satellite during the 7-second tumble cycle. Such a change prevents the longitudinal magnetic torques from averaging to zero due to rotational symmetry. Using the 


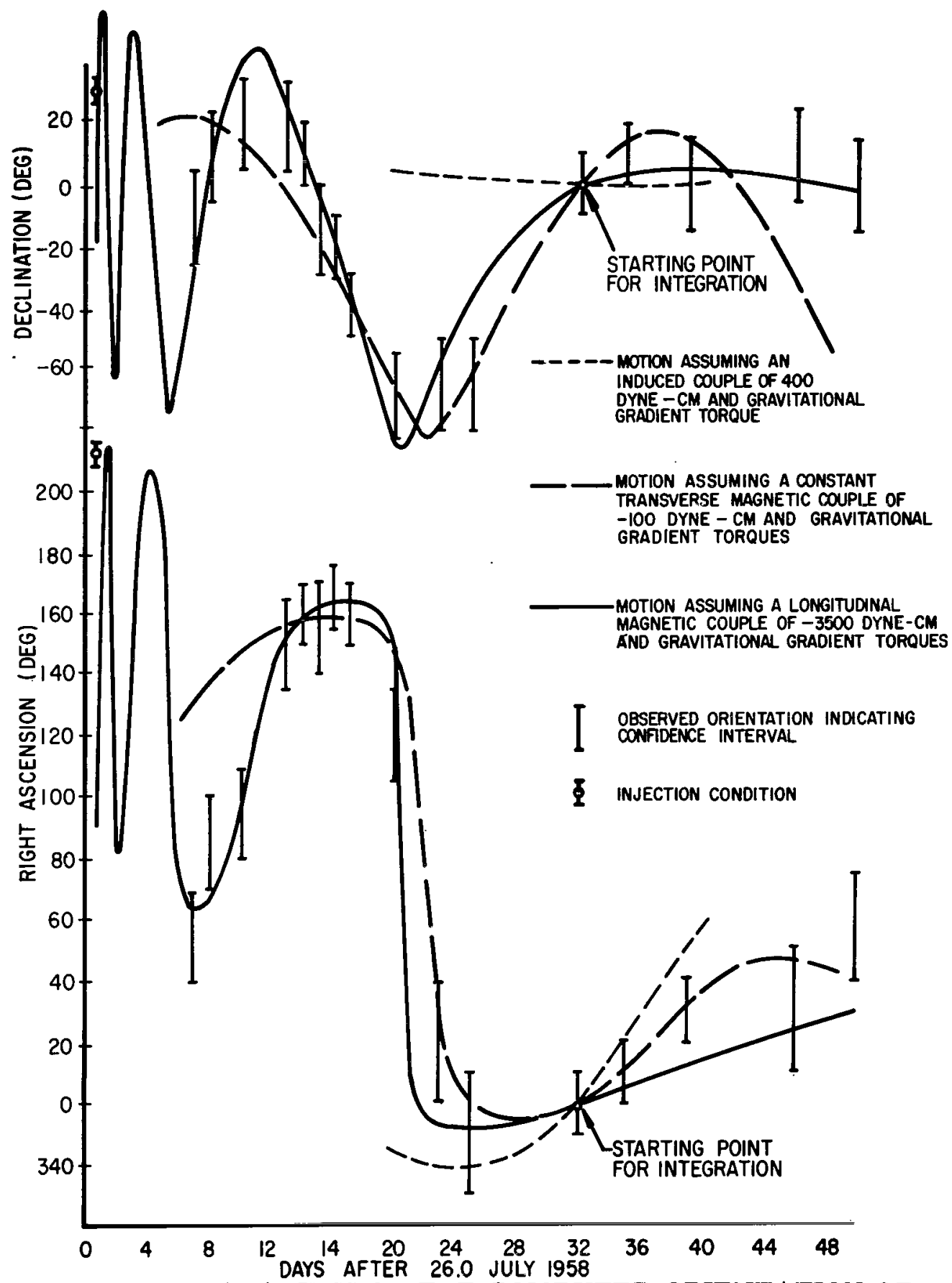

FIGURE 6. ANALYSIS OF THE COMPUTED ORIENTATION OF EXPLORER IV DUE TO VARIOUS ASSUMPTIONS COMPARED TO OBSERVATION 


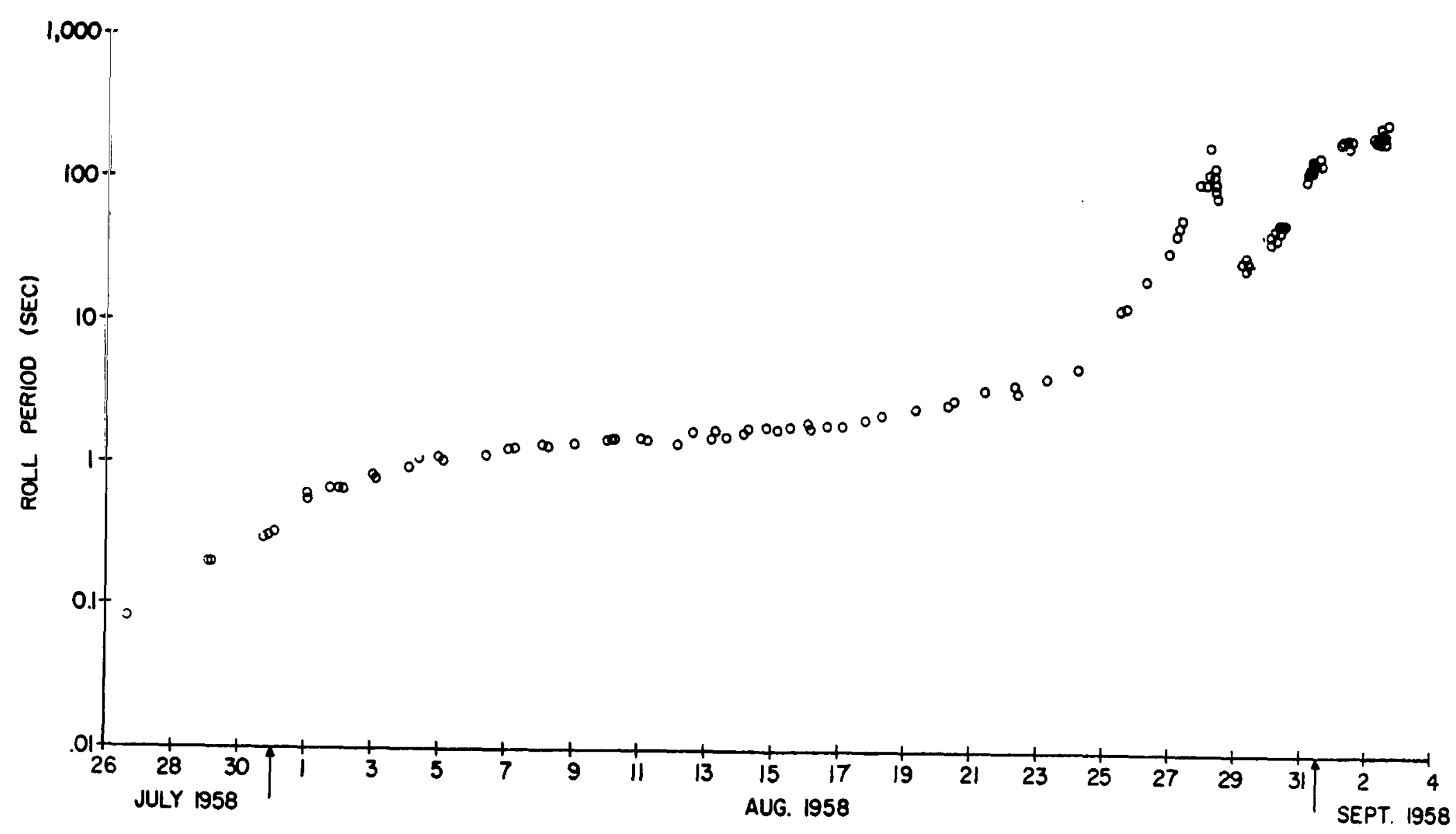


formulation of the full six degree of freedom problem discussed in the introduction, it was found that the net second-order effect was approximately $10^{-3}$ of the maximum instantaneous magnetic torque. This effect would therefore become significant only if there exists a large magnetic moment having rotational symmetry and if the other torque-producing effects are small.

Some justification is necessary for the fact that a couple of -3500 dyne-cm corresponding to a longitudinal moment of $19 \mathrm{amp}-\mathrm{m}^{2}$ is required to explain Explorer IV motion, whereas only 450 dyne-cm or $2.35 \mathrm{amp}-\mathrm{m}^{2}$ is required for Explorer XI. This can be resolved, qualitatively at least, by the fact that the instrumented portion of Explorer $\mathrm{IV}$ is stainless steel rather than aluminum. Furthermore, this portion was placed on a magnetically driven shake table for vibrational testing prior to flight. This quite probably resulted in a rather large permanent magnetization. Taking the volume of ferrous material to be $450 \mathrm{~cm}^{3}$, a flux density of approximately 500 gauss in the material is required to produce a magnetic moment of $19 \mathrm{amp}-\mathrm{m}^{2}$. The saturation of 410 stainless steel is on the order of 16 kilogauss and the retentivity is approximately 8 kilogauss for 10 kilogauss applied. Hence, 500 gauss is well within the magnetic capabilities of the material.

It is curious that Explorer IV began tumbling very shortly after injection but required 30 or more days to approach $\pi / 2$. The longitudinal roll $\omega_{3}$ apparently never reached zero and, in fact, even appears to increase on day 33. This increase is possi. bly due to an interaction torque from a transverse magnetic moment. On the other hand, Explorer XI had a very small half-cone angle until about day 20, at which time it opened rapidly and lost all its $\omega_{3}$ within a few days. These facts may be explained by the consideration that Explorer IV did not have a mercury damper and the dissipative forces became less effective in removing energy as $\omega_{3}$ became small. It is difficult to understand why Explorer XI, with a mercury damper, took so long to begin tumbling. There is evidence that a tape recorder inside the satellite was accidentally commanded on and left running after launch. There is also evidence that this recorder failed on about the day the tumbling started. It has been suggested that the recorder, through bearing friction, actually put rotational energy into the satellite which overcame the energy dissipated by mechanical flexing [Ref. 14]. This presumably allowed the satellite to remain in its maximum energy configuration despite dissipative losses.

\section{SECTION V. EXPLORER VIII}

Unlike the satellites discussed previously, Explorer VIII was of a stable configuration. No attempt was made to analyze the orientation by radio signal strength techniques, but the satellite contained a crossed-slit solar aspect angle measuring device along with a horizon sensor. Presumably the data from both of these devices would determine the orientation of the spin axis. The solar aspect device yielded excellent 
data, accurate to about 1 degree. However, the horizon scan data a re apparently difficult to interpret. The only published orientation data are the angles between the spin axis and the sun for the first 30 days [Ref. 15].

From the previous analysis, it was felt that the torques acting on Explorer VIII must be predominately due to a permanent magnetic moment along the spin axis and gravitational torques. Therefore, an attempt was made to integrate the motion from the injection condition to produce the observed solar aspect angle.

The first measured solar aspect angle did not agree with an assumed initial spin axis coinciding with the injection velocity vector; hence, it was necessary to assume a spin axis injection condition a few degrees from the velocity vector at injection. This can be reconciled by a slight misalignment of the fourth stage velocity vector with the total injection velocity, or by assuming that the satellite was precessing slightly when the fourth stage was jettisoned.

Various values of $M_{3} B_{N}$ were assumed until it was found that $M_{3} B_{N}=-200$ dyne-cm .would produce a computed solar aspect angle shown in Figure 8. This computed result compares with the measured solar aspect angle well within the limits of accuracy. The resulting trace of the spin axis is shown in Figure 9.

\section{SECTION VI. EXPLORER VII}

A crude determination of Explorer VII orientation was accomplished by noting the times at which the fading caused by Faraday rotation of the $20 \mathrm{mc} / \mathrm{sec}$ signal strength became the most severe [Ref. 16]. The turnstile antenna emits omnidirectional radiation that is circularly polarized along the spin axis. The polarization becomes more elliptical as the angle from the spin axis is increased, becoming linearly polarized in the plane of the equator. Since the Faraday fading becomes the most severe for a linearly polarized wave, the observance of this severe fading indicates the station to satellite vector is normal to the spin axis.

During the first two months, the orientation appeared to remain almost constant at the injection condition. A slight shift was indicated, but the limits of accuracy of the measurement were not sufficient to ascertain this. Thermal measurements taken throughout the first year of orbiting were in general agreement with the assumption of a space-fixed orientation, although again such measurements are not sensitive enough to detect a slight shift of the spin axis [Ref. 16].

It is certain that gravitational torques act on Explorer VII and it seems virtually impossible to construct a satellite without some magnetic moment unless particular 


\section{ANGLE SPIN AXIS MAKES WITH SUN}

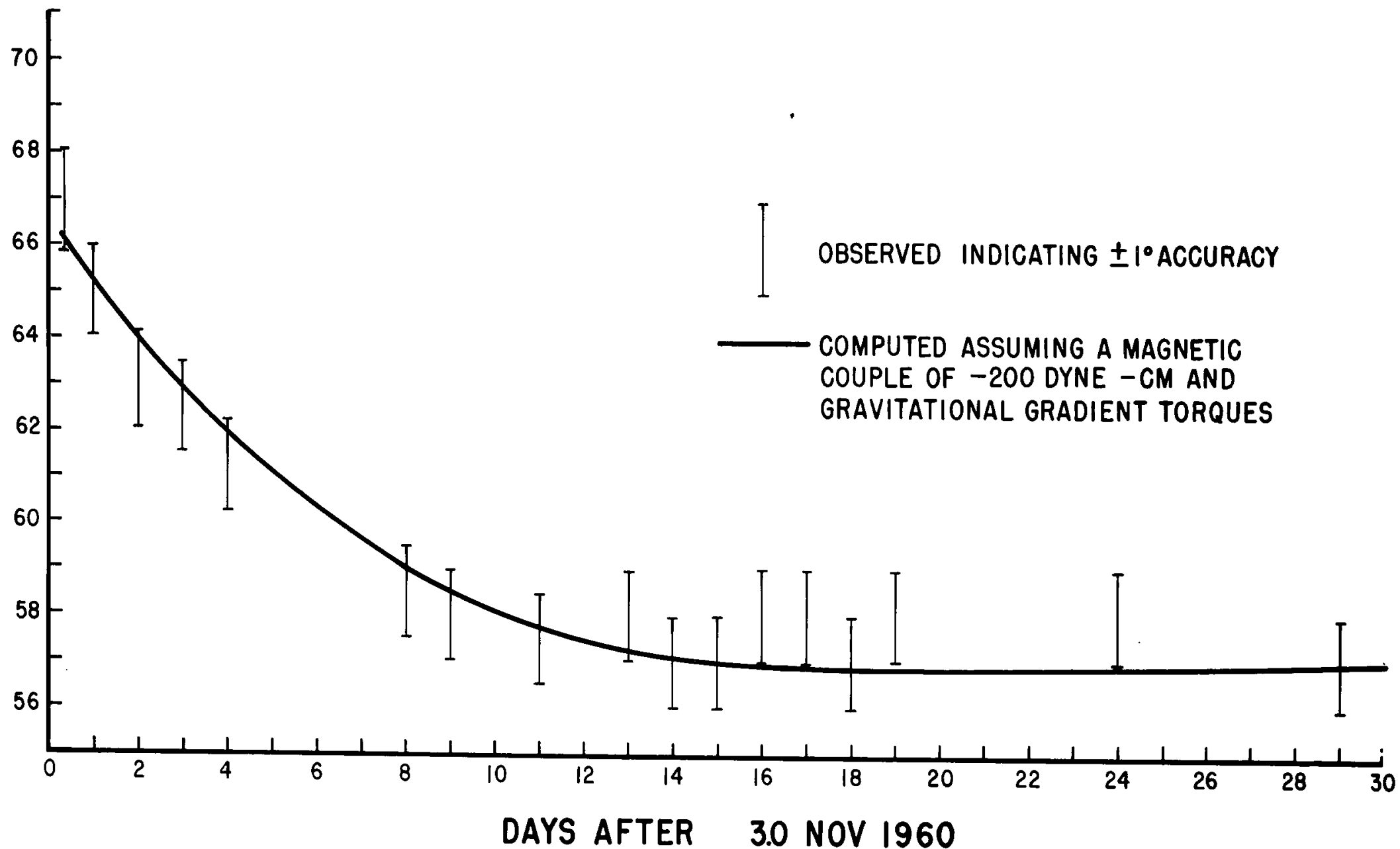

FIGURE 8. COMPUTED SOLAR ASPECT ANGI.F FOR EXPLORER VIII COMPARED TO OBSERVATIONS 


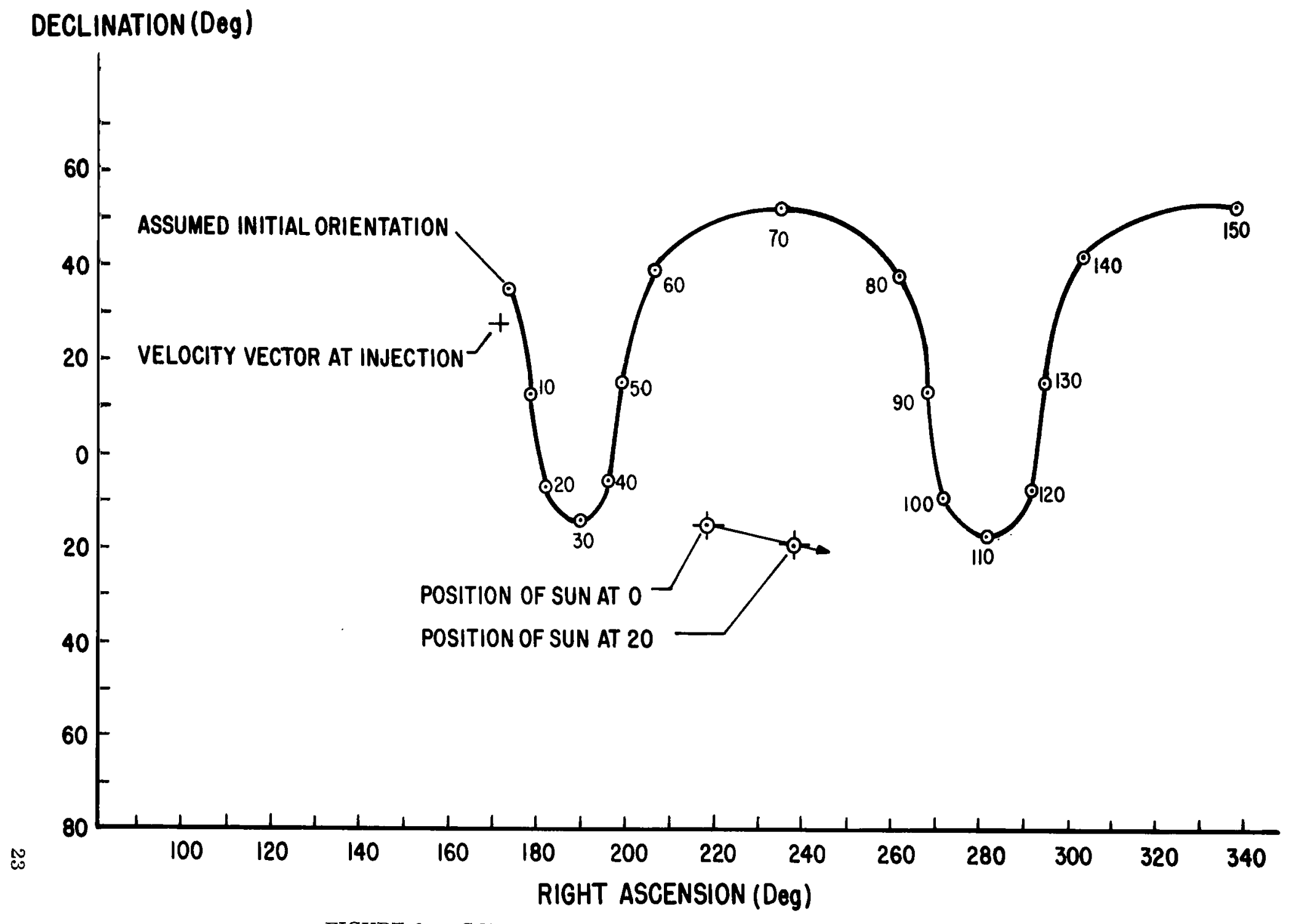

FIGURE 9. COMPUTED ORIENTATION OF EXPLORER VIII 
care is taken to prevent such a moment . Explorer VII does have a very large angular momentum resulting from its fast spin rate which greatly reduces the forced precession of the spin axis.

The motion of the spin axis was integrated, assuming only gravitational torques, and resulted in the dashed curve in Figure 10. This is seen to result in a very slow net drift of the spin axis with time. The addition of a small magnetic moment with $\mathrm{M}_{3} \mathrm{~B}_{\mathrm{N}}=$ 75 dyne-cm produces a motion indicated by the solid line. Again, it is seen that although an oscillation of several degrees is present, the net drift is quite small. This motion also appears to fit the observed orientation data, although the accuracy is not really sufficient to confirm it.

\section{SECTION VII. CONCLUSIONS}

It now seems clear that permanent magnetic and gravitational torques are the dominating effects which produce changes in a satellite orientation. Much the same conclusion was reached by Manger [Ref. 17]. for the Tiros satellites. In fact, the orientation of Tiros III was effectively controlled by applying currents in a loop to alter the magnetic moment. The orientation was predicted and the control currents were programmed prior to launch. According to Hetch and Manger [Ref. 18], this was quite successful. Other satellites have been passively oriented along the magnetic field lines by employing permanent magnets and hysteresis damping rods [Ref. 19].

The magnetic effects in Explorer XI and Explorer IV were not as obvious as those in stable spinning satellites since it was not clear that a permanent magnetic moment projection on the axis of rotation existed. But now that the motions of these satellites have been successfully analyzed and understood, a heretofore disturbing problem has been resolved.

It is now felt that sufficient knowledge of the rotational behavior of satellites exists to make it worthwhile to formulate the equations of motion about the center of mass with those for the orbit and solve the full six degree of freedom equations of motion. Particular care should be taken to determine the moments of inertia and magnetic moments prior to flight.

It was also shown that simplifying approximations may be employed that give a fairly good representation of the motion without integrating the orbital equations. The orbital information necessary to use these approximate equations is the semi-major axis, the inclination, and right ascension of the ascending node. These equations are simple enough to be integrated on a small computer such as a ReComp II or LGP-30 or, if necessary, by hand. 

Knowledge of the torque-producing effects is also of vital importance in active attitude-controlled satellites since such information can be used to determine the optimum saturation level for inertial devices and propellant storage requirements for mass expulsion devices. 


\section{REFERENCE}

1. Lundquist, C. A. and Naumann, R. J., "Orbital and Rotational Motion of a Rigid Satellite," Seminar Proceedings; Tracking and Orbit Determination, Jet Propulsion Laboratory, Cal. Inst. of Tech., Pasadena, California, 1960.

2. Whitaker, E. T. , A Treatise on the Analytical Dynamics of Particles and Rigid Bodies, Fourth Edition, Dover Publishing Company, 1944.

3. Goldstein, H. , Classical Mechanics, Addison-Wesley Publishing Company, Inc., Cambridge, Mass., 1950.

4. Roberson, R.E. and Tatistcheff, D. , "The Potential Energy of a Small Rigid Body in the Gravitational Field of an Oblate Spheroid," J. Franklin Inst. , Vol. 262, pp. 209-214, 1956.

5. Doolin, B. F., "Gravity Torque on an Orbiting Vehicle," NASA TN D-10, Ames Research Center, Moffet Field, California, 1960.

6. Colombo, G. , "On the Motion of Explorer XI Around its Center of Mass," American Astronautical Society, Goddard Memorial Symposium, Washington, D. C. March 16-17, 1962.

7. Finch, H. F., and Leaton, B. R. "The Earth's Main Magnetic Field, Epoch 1955. 0," Mon. Notices of the Royal Astronomical Society, 7, pp. 313-317, 1957.

8. Naumann, R. J., Fields, S. A., and Holland, R. L., "Analysis of Explorer XI Orientation," J. Geophys. Research, 67, pp. 3619-3623, 1962.

9. Naumann, R.J., "Recent Information Gained from Satellite Orientation Measurement," Plan. and Space Sci., Vol. 7, pp. 445-453, 1961.

10. Lundquist, C.A., Naumann, R. J., and Fields, S. A., "Recovery of Further Data from 1958 Epsilon," Space Research, II (ed. by van de Hulst, Jager, and Moore), N. Holland Pub. Co., Amsterdam, 1961.

11. Lundquist, C. A., Naumann, R. J., and Weber, A. H., "Directional Flux Densities and Mirror-Point Distributions of Trapped Particles from Satellite 1958 Epsilon Measurements," J. Geophys, Research, 67, pp. 4125-4133, 1962. 
12. Colombo, G., "The Motion of Satellite 1958 Epsilon Around Its Center of Mass," Research in Space Science, Special Report No. 70, Smithsonian Institution Astrophysical Observatory, Cambridge, Mass. , July 18, 1961.

13. Fields, S. A., "Body Motions of 1958 Epsilon," Army Ballistic Missile Agency Tech. Memo. CL-TM-11-60, 1960.

14. Kuebler, M.E., "Spin-Tumble Transfer of Satellite S-15 (Explorer XI)," Marshall Space Flight Center MTP-G\& C-61-30, 1961.

15. Bourdeau, R.E., Donley, J.L., and Whipple, E.C. , "The Ionosphere Direct Measurements Satellite Instrumentation (Explorer VIII)," National IAS-ARS Joint Meeting, Los Angeles, Calif. June 13-16, 1961.

16. Heller, G. B., Jones, B. P. , Naumann, R. J. and Snoddy, W. C. , "Correlation of the Thermal Behavior of Satellites and the Rotational Momentum Vector," From Peenemunde to Outer Space, (edited by E. Stuhlinger, F. Ordway, III, J. McCall, and G. Bucher) Marshall Space Flight Center, Huntsville, Alabama, pp. 203-240, March 23, 1962.

17. Bandeen, W. R. and Manger, W. P., "Angular Motion of the Spin Axis of Tiros I Meteorological Satellite Due to Magnetic and Gravitational Torque," J. Geophys. Res. 65, pp. 2992-6, 1960.

18. Hetch, E. and Manger, W. P., "Magnetic Attitude Control of the Tiros Satellites," American Astronautical Society, Goddard Memorial Symposium, Washington, D. C. , March 16-17, 1962.

19. Fischell, R.E. , "Passive Magnetic Attitude Control for Earth Satellites," American Astronomical Society, Goddard Memorial Symposium, Washington, D. C. , March 16-17, 1962. 
"The aeronautical and space activities of the United States shall be conducted so as to contribute. . . to the expansion of buman knowledge of phenomena in the atmosphere and space. The Administration shall provide for the widest practicable and appropriate dissemination of information concerning its activities and the results thereof."

-National Aeronautics and Space Act of 1958

\section{NASA SCIENTIFIC AND TECHNICAL PUBLICATIONS}

TECHNICAL REPORTS: Scientific and technical information considered important, complete, and a lasting contribution to existing knowledge.

TECHNICAL NOTES: Information less broad in scope but nevertheless of importance as a contribution to existing knowledge.

TECHNICAL MEMORANDUMS: Information receiving limited distribution because of preliminary data, security classification, or other reasons.

CONTRACTOR REPORTS: Technical information generated in connection with a NASA contract or grant and released under NASA auspices.

TECHNICAL TRANSLATIONS: Information published in a foreign language considered to merit NASA distribution in English.

TECHNICAL REPRINTS: Information derived from NASA activities and initially published in the form of journal articles.

SPECIAL PUBLICATIONS: Information derived from or of value to NASA activities but not necessarily reporting the results of individual NASA-programmed scientific efforts. Publications include conference proceedings, monographs, data compilations, handbooks, sourcebooks, and special bibliographies.

Details on the availability of these publications may be obtained from:

SCIENTIFIC AND TECHNICAL INFORMATION DIVISION

NATIONAL AERONAUTICS AND SPACE ADMINISTRATION

Washington, D.C. 20546 